Impact of early azole therapy on chronic colonisation with Aspergillus fumigatus during cystic fibrosis

JP Gangneux ${ }^{1}$, A Horsley², C Belleguic ${ }^{1}$, E Deneuville 1 , S Chevrier ${ }^{1}$, B Laviolle ${ }^{1}$, S Leroy ${ }^{3}$, L Delhaes ${ }^{3}$, D Denning ${ }^{2}$ ${ }^{1}$ Rennes University Hospital, France, 2University Hospital of South Manchester, UK, ${ }^{3}$ Lille University Hospital, France.

Objectives. Early azole therapy during cystic fibrosis (CF) remains debated: when to start ? with which azole ? when to stop ? The ATCF (Antifungal Therapy during Cystic Fibrosis) is an exploratory longitudinal phase III study with the following objectives:

the primary objective was to assess the effect of azole therapy on the negativation of sputum cultures for Aspergillus on 2 consecutive samples. Secondary objectives were to compare the course and outcome of FEV1, clinical signs and quality of life, the impact on co-prescription of antibiotic courses and steroid therapies, the speed of negativation of sputum cultures for Aspergillus, the efficacy of treatments on outcome of other diagnostic criteria (Aspergillus precipiting lgG, total and specific lgE, eosinophil counts), and safety profiles of the azoles.

Methods. The ATCF study followed 11 patients presenting with a chronic colonisation with $A$. fumigatus and randomized to receive an early azole therapy for 4 to 6 months in a phase III study. Treatments evaluated were itraconazole (ITRA) oral solution or voriconazole (VORI) oral suspension at the following regimens: $2 \times 200 \mathrm{mg} /$ day for patients $>40 \mathrm{~kg}$ and $2 \times 100 \mathrm{mg} /$ day for patients $<40 \mathrm{~kg}$. A long-term follow-up was conducted until 2 months after end of treatment.

\section{Ethics Statement}

Written informed consent on the use of the anonymized epidemiological, clinical and prospective clinical data was obtained from each patient or their guardian for those aged under 18. Clearance was obtained from the ethical committees of Rennes, Lille and Manchester hospitals.

Results. Six patients were included in the ITRA arm and 5 in the VORI arm. The Delta F508 mutation was present in $91 \%$ of the patients; the mean predicted FEV1 at inclusion was of $50.39 \% \pm 18.01$

$(44.22 \% \pm 14.71$ in ITRA arm and $57.79 \% \pm 20.36$ in the VORI arm). Four patients stopped treatments (1 ITRA and 3 VORI) prematurely because of intolerance. Seven/7 (100\%) assessable patients had a successfull outcome, with a negativisation of sputums after 4 to 6 months of treatment (5/5 ITRA et $2 / 2$ VORI). Increase in FEV1 was observed as soon as 4 weeks after treatment initiation for $6 / 9$ patients, from $50.39 \% \pm 18.01$ to $63.30 \% \pm 30.21$. The number of patients not receiving antibiotics grew from $18.2 \%$ at day0 to $82 \%$ and $89 \%$ at weeks 2 and 4 , respectively. Aspergillus precipiting IgG as well as total and specific IgE also significantly decreased. However, 2 months after the end of treatment, most of assessment criteria were similar to the baseline values.
Figure 1. Flow chart of inclusion

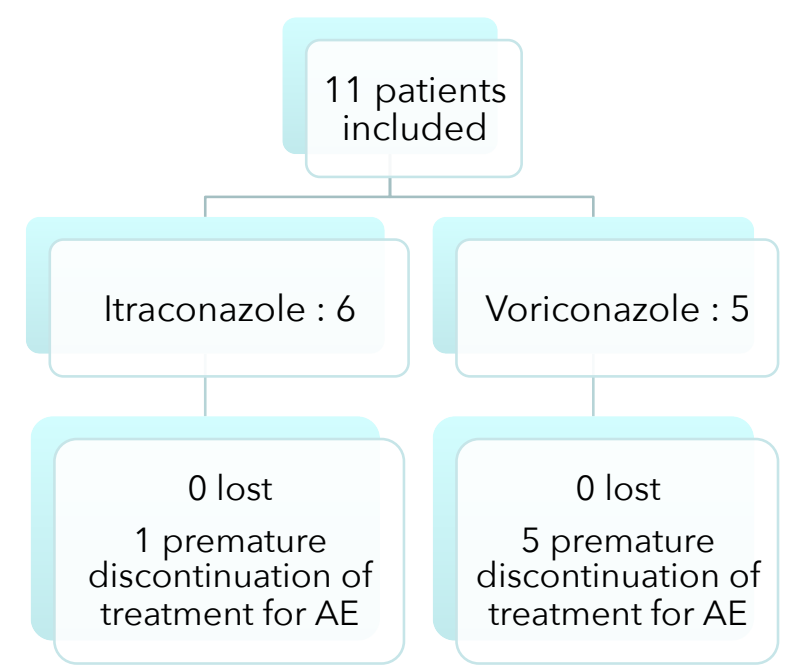

Inclusions. Eleven patients were included, 6 men and 5 women, 9 adults and 2 children, from Rennes $(\mathrm{Fr})$, Lille (Fr) and Manchester (UK) hospitals. The ITRA arm consisted of 2 men and 4 women, all adults. The VORI arm consisted of 4 men and 1 woman and 3 adults and 2 children.

Pharmacology. Dose adjustment at day 15 was required for $70 \%$ of patients: 2 dose increases for 5 ITRA (40\%, mean $8.98 \mathrm{mg} / \mathrm{L} \pm 10.87$ ) and $5 / 5$ (100\%, 4 increases, 1 stop, mean rate $0.40 \mathrm{mg} / \mathrm{L} \pm$ $0.23)$ VORI. Adjusted rates were $5.01 \mathrm{mg} / \mathrm{L} \pm 4.65$ for ITRA and $1.41 \mathrm{mg} / \mathrm{L} \pm 1.62$ for VORI.

Figure 2. Results of primary and secondary endpoints

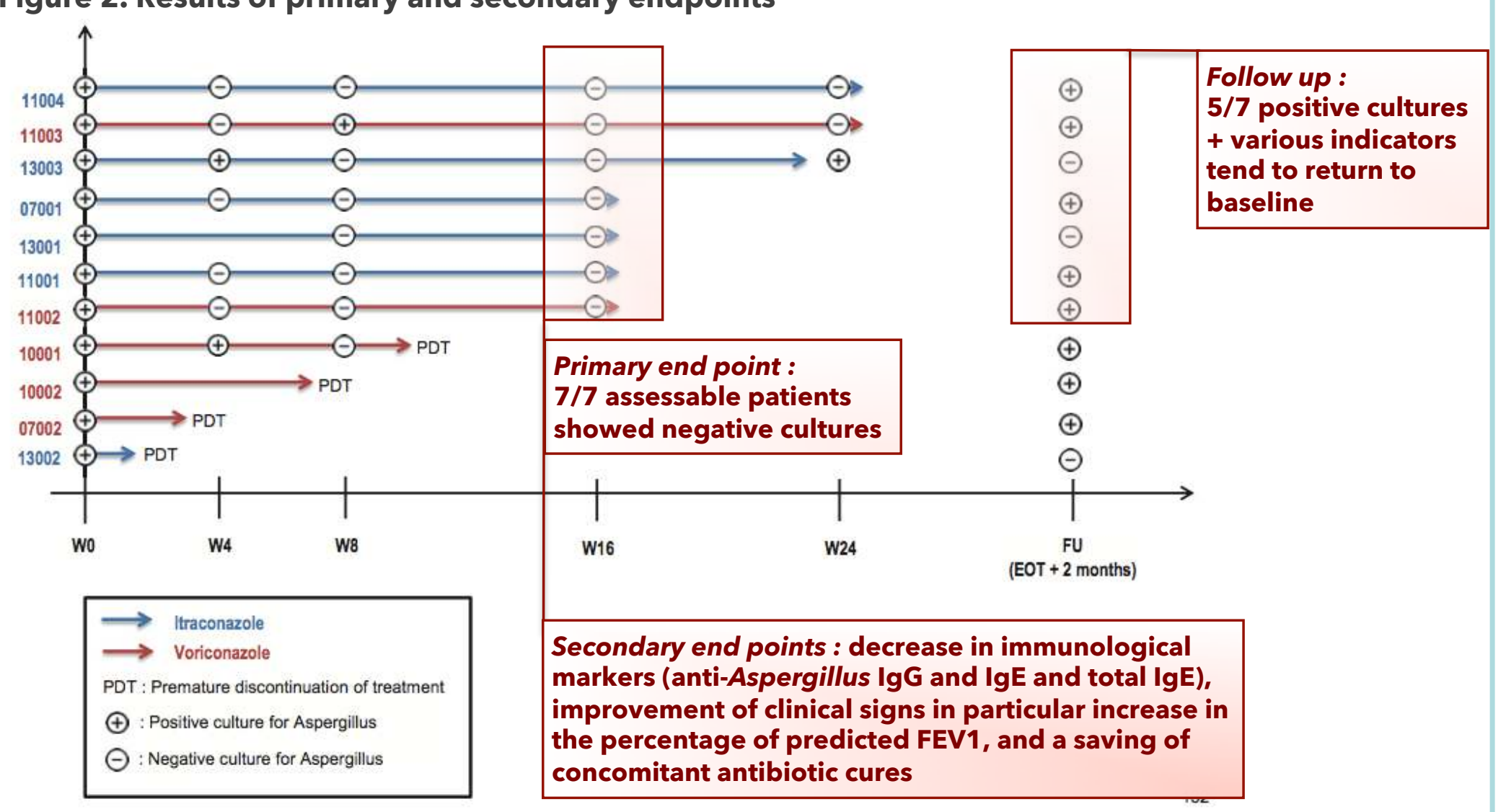

Figure 3. FEV1 evolution under treatment

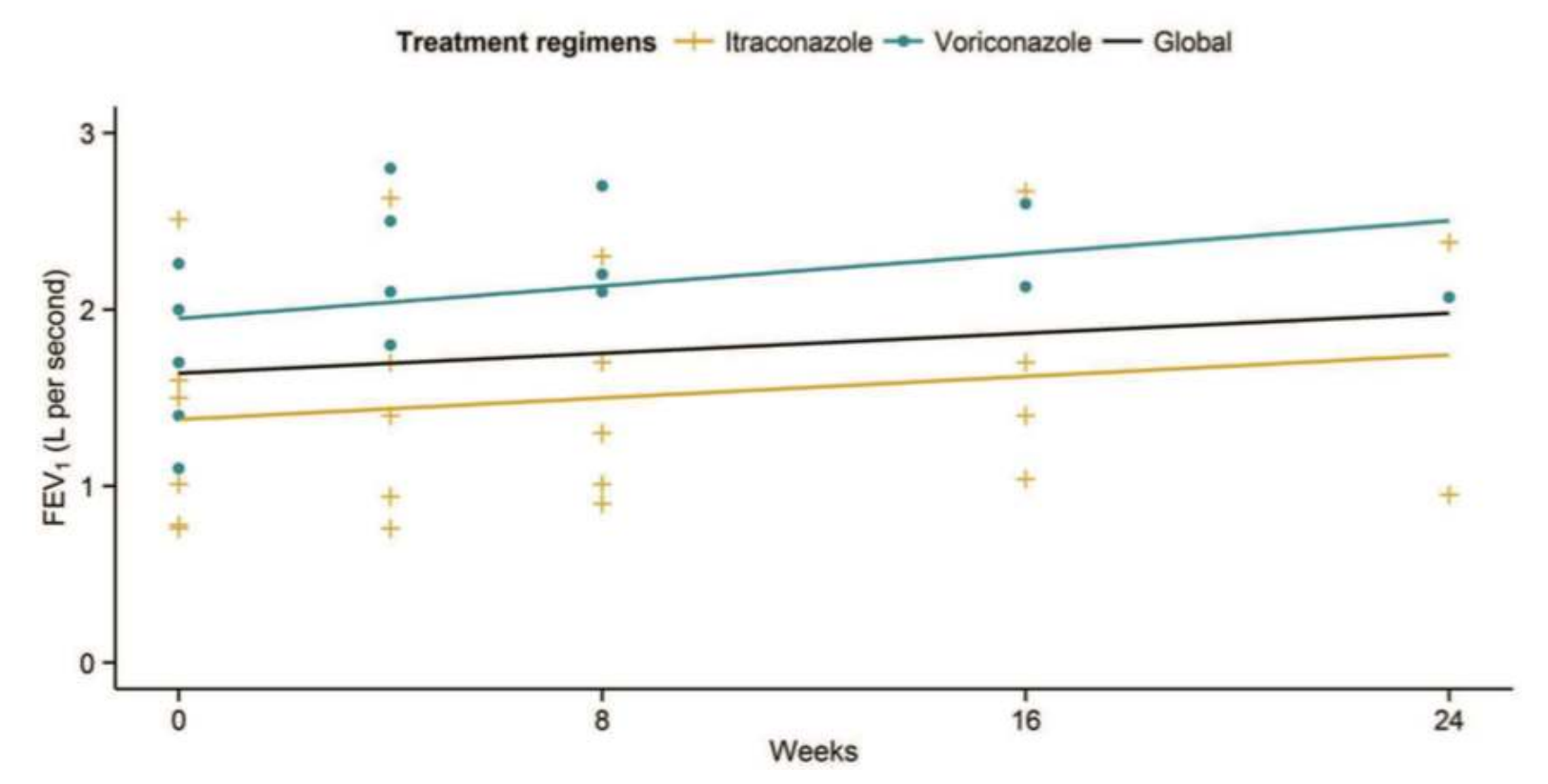

Figure 4. Saving of antibiotic cures (\% of patients without antibiotics)

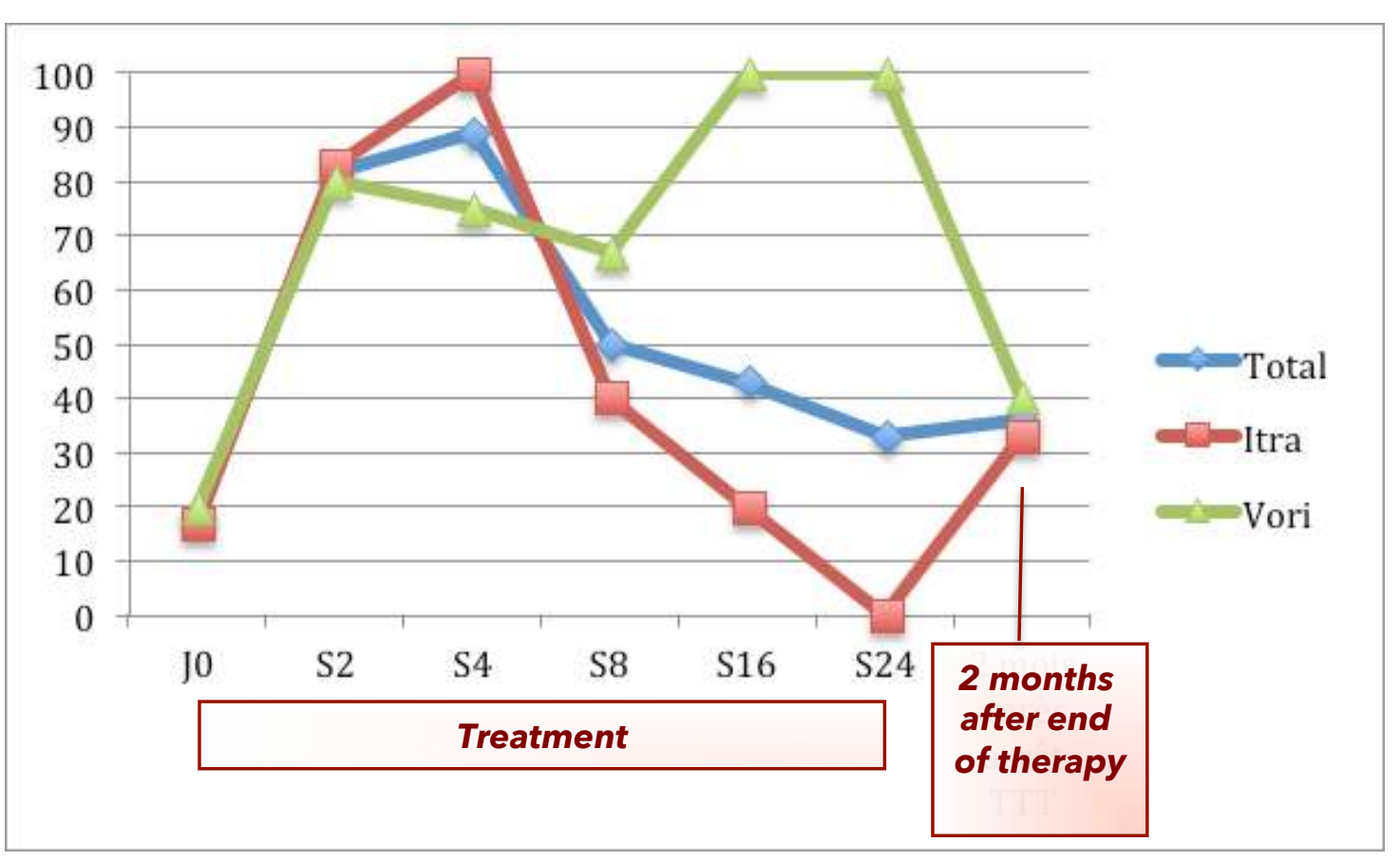

Conclusion. While only a limited number of patients were included, the strict and meticulous longitudinal phase III design of ATCF study underlines the need for azole therapy rationalisation during CF. When a chronic colonisation with $A$. fumigatus is associated with clinical signs, early azole therapy can temporarily reduce airway infection and inflammation, thus extending the periods of stable disease status and stable lung function with a positive collateral consequence of antibiotic savings.

These data highlight the value of rationalizing the prescription of azole antifungals during cystic fibrosis by measuring well the benefit / risk ratio. When the need is objectified and encourages prescription, it is imperative to closely monitor tolerance and monitor serum levels to adjust the dose. The efficacy being rapid on both clinical and biological markers but not perennial, it is desirable to favor short cures to manage the acute episode and then to give the means to stop the treatment to avoid iatrogeny and resume if needed.

These data will be complemented by more fundamental studies under investigation: the evolution of the microbiome and the inflammasome of cystic fibrosis patients under azole therapy, using the most modern techniques of massive sequencing and DNA microarray. 